\title{
MiR-219-5p inhibits growth and metastasis of ovarian cancer cells by targeting HMGA2
}

\author{
Feng Xing, Zhijiao Song and Yuanying $\mathrm{He}^{*}$
}

\begin{abstract}
Background: Accumulating studies have demonstrated that high-mobility group A2 (HMGA2), an oncofetal protein, plays a role in tumor development and progression. However, the molecular role of HMGA2 in ovarian carcinoma is yet to be established. MicroRNAs (miRNAs), a group of small noncoding RNAs, negatively regulate gene expression and their dysregulation has been implicated in tumorigenesis. The aim of this study was to investigate the potential involvement of a specific miRNA, miR-219-5p, in HMGA2-induced ovarian cancer.

Methods: The ovarian cancer cell line, SKOV3, was employed, and miR-219-5p and HMGA2 overexpression vectors constructed. The CCK-8 kit was used to determine cell proliferation and the Transwell ${ }^{\circledR}$ assay used to measure cell invasion and migration. RT-PCR and western blot analyses were applied to analyze the expression of miR-219-5p and HMGA2, and the luciferase reporter assay used to examine the interactions between miR-219-5p and HMGA2. Nude mice were employed to characterize in vivo tumor growth regulation.
\end{abstract}

Results: Expression of miR-219-5p led to suppression of proliferation, invasion and migration of the ovarian cancer cell line, SKOV3, by targeting HMGA2. The inhibitory effects of miR-219-5p were reversed upon overexpression of HMGA2. Data from the luciferase reporter assay showed that miR-219-5p downregulates HMGA2 via direct integration with its 3'-UTR. Consistent with in vitro findings, expression of miR-219-5p led to significant inhibition of tumor growth in vivo.

Conclusion: Our results collectively suggest that miR-219-5p inhibits tumor growth and metastasis by targeting HMGA2.

Keywords: Ovarian cancer, miR-219-5p, HMGA2, Growth, Metastasis

\section{Background}

Ovarian cancer has the highest mortality rate of all gynecologic neoplasms and is the fifth leading cause of cancer-related death in females in Western countries [1, 2]. Although remarkable advances have been made in the treatment of this tumor type [3], the long-term prognosis of metastatic melanoma remains poor.

High-mobility group A2 (HMGA2) is a small nuclear protein belonging to the high mobility group (HMG)

\footnotetext{
*Correspondence: haze19841224@me.com

${ }^{\dagger}$ Feng Xing and Zhijiao Song are co-first author Department of Obstetrics and Gynecology, Shanghai Tenth People's Hospital of Tongji University, Tongji University School of Medicine, No 301 Middle Yan Chang Road, Shanghai 200072, China
}

family. The protein modulates multiple genes through effects on protein-DNA or protein-protein interactions $[4,5]$. HMGA2 has been identified as an oncoprotein that is frequently upregulated in a variety of cancers, including breast cancer [6], esophageal squamous carcinoma [7], colorectal cancer [8, 9], ovarian tumors [10]. However, the mechanisms underlying regulation of HMGA2 in ovarian tumors are currently unclear.

MicroRNAs (miRNAs) are small noncoding RNAs 15-22-nucleotides in length [11] proposed to be correlated with tumor growth and metastasis based on their protumor or antitumor effects in a number of neoplasms [12]. Recent bioinformatics analyses (http://www.genec ards.org/) have revealed that miR-219-5p targets the 
3'UTR of HMGA2. Abnormal expression and tumor suppressor activity of miR-219-5p in different malignant cancer types have been documented in the literature [13, 14]. However, the role of miR-219-5p in ovarian cancer remains unclear at present.

The main aim of this investigation was to establish whether miR-219-5p-HMGA2 interactions play a regulatory role in ovarian cancer progression. Our results showed that increased expression of miR-219-5p leads to significant reversal of HMGA2-induced cell migration, invasion and proliferation of ovarian cancer cells. In addition, miR-219-5p was determined as a regulator that inhibits HMGA2 expression in ovarian cancer.

\section{Materials and methods}

\section{Ethics statement}

All animal experiments were approved by the Ethics Committee of Shanghai Tenth People's Hospital, Shanghai, China. Surgical procedures were performed under anesthesia and every effort was made to minimize suffering of animals. All mice were anesthetized via intraperitoneal injection of sodium pentobarbital $(30 \mathrm{mg} / \mathrm{kg})$.

\section{Cell lines and cultures}

Both SKOV3 and HEK293T cell lines were obtained from the American Type Culture Collection (Manassas, VA, USA). SKOV3 cells were cultured in RPMI 1640 (Invitrogen, California, USA), and HEK293T in Dulbecco's Modified Eagle Medium (Invitrogen, California, USA) supplemented with $10 \%$ fetal bovine serum (FBS; Invitrogen, California, USA) at $37^{\circ} \mathrm{C}$ in $5 \% \mathrm{CO}_{2}$.

\section{Transfection of cells with miR-219-5p mimic or HMGA2 overexpression vector}

The miR-219-5p mimic vector was synthesized by GenePharma (Shanghai, China). Full-length HMGA2 from the human cDNA library was cloned into pCDNA3.1 vector. Cells were transfected using the Lipofectamine 2000 reagent (Thermo Scientific, Massachusetts, USA) according to manufacturer's instructions.

\section{Qualitative PCR analysis}

Total RNA was extracted from tissues and cells using TRIzol reagent (Invitrogen, California, USA) in keeping with the reagent kit protocol and cDNA amplified using the TaqMan miRNA reverse transcription kit (Invitrogen, California, USA). HMGA2, miR-219-5p and U6 mRNA levels were determined via $\mathrm{qPCR}$ using the TaqMan human miRNA assay kit. Relative fold difference was measured using the $2^{-\triangle \Delta C T}$ method. The following primers were employed: HMGA2 (F) 5'-CTCAAAAGAAAG CAGAAGCCACTG- $3^{\prime}$ and (R) 5'-TGAGCAGGCTTC TTCTGAACAACT-3'; miR-219-5p (F) 5'-ACACTC
CAGCTGGGTGATTGTCCAAACGCAAT- $3^{\prime}$ and (R) 5'-CTCAACTGGTGTCGTGGA-3'; U6 (F) 5'-CTCGCT TCGGCAGCACA-3' and (R) 5'-AACGCTTCACGA ATTTGCGT-3'.

\section{CCK-8 assay}

Cell proliferation was detected with the CCK- 8 assay according to the manufacturer's protocol (Invitrogen, California, USA). SKOV3 cells from different groups were cultured in 96-well plates under the same conditions. After 0, 24, 48 and $72 \mathrm{~h}$, a mixture of $90 \mu \mathrm{l}$ fresh culture medium and $10 \mu \mathrm{l}$ CCK- 8 solution was added to wells. Following further incubation of SKOV3 cells at $37{ }^{\circ} \mathrm{C}$ for $2 \mathrm{~h}$, cells were examined using a microplate reader at a wavelength of $450 \mathrm{~nm}$.

\section{Luciferase reporter assay}

HMGA2 with wild-type or mutant $3^{\prime}$-UTR was generated and cloned into the firefly luciferase-expressing vector, psiCHECK-2. For the luciferase assay, cells were seeded in triplicate in 12-well plates the day before transfection and subsequently transfected with WT or Mut 3'-UTR reporter vector, either in combination with or without the miR-219-5p mimic. Next, cells were harvested and lysed for luciferase activity analysis using the Dual-Luciferase Reporter System (Promega, WI, USA). Three independent experiments were performed.

\section{Western blot assay}

Cells or tissues were lysed, protease inhibitors added to the lysates and centrifuged at $12,000 \mathrm{rpm}$ at $4{ }^{\circ} \mathrm{C}$. Protein concentrations were examined with the BCA kit (Pierce, USA). Next, proteins were separated via $10 \%$ SDS-PAGE and transferred to PVDF membranes. The following antibodies were employed for detection of protein expression: HMGA2 (1:500, Santa Cruz, California, USA) or anti-GAPDH (1:2000, Santa Cruz, California, USA) primary antibodies and horseradish peroxidase-conjugated secondary antibody (1:1000, Santa Cruz, California, USA). The ECL chemiluminescent kit (Millipore, MA, USA) was applied to visualize protein bands.

\section{Transwell assay}

Cells were transfected with miR-219-5p mimic or HMGA2 overexpression vector or pretreated with miR219-5p inhibitor. After $48 \mathrm{~h}$, cells were cultured in serum deprivation medium for $12 \mathrm{~h}$ and digested with trypsin before seeding on the top chambers of 24-well transwell culture inserts (Promega). Medium supplemented with $20 \%$ serum was used as a chemoattractant in the lower chambers. After $24 \mathrm{~h}$, cells were fixed for 10 min with $4 \%$ formalin before staining with $0.005 \%$ crystal violet and counted under a phase contrast microscope. 
Invasion assays were performed using the BD BioCoat Matrigel invasion assay system (BD Biosciences, NJ, USA) according to the manufacturer's instructions. The non-motile or noninvasive cells were removed while the lower side of the filter was stained with $0.005 \%$ crystal violet and counted.

\section{Tumor growth in vivo}

After transfection, $2 \times 10^{6} \mathrm{SKOV} 3$ cells were transplanted into nude mice (25-30 g, 6 weeks old, $n=6$ ). Gross tumor volumes were measured with vernier calipers every 5 days from days 5 to 25 . Mice were subsequently killed for western blot analysis.

\section{Statistical analysis}

Continuous variables were expressed as mean \pm standard deviation (SD). One-way ANOVA was performed for multiple comparisons using GraphPad Prism software, version 5.0 (GraphPad, La Jolla, CA, USA). P-values $\leq 0.05$ indicated statistically significant differences.

\section{Results}

miR-219-5p overexpression suppresses ovarian carcinoma cell proliferation, migration and invasion

To establish the specific role of miR-219-5p, SKOV3 cells were transfected with an miR-219-5p overexpression vector (miR-219-5p mimic) or pretreated with an miR219-5p inhibitor for 48 h. qRT-PCR results revealed a significant increase in miR-219-5p expression after transfection with the miR-219-5p mimic and conversely, significant suppression of miR-219-5p following treatment with a specific inhibitor, compared with the scramble group (Fig. 1a). Cell proliferation was determined with a cell counting kit using an initial concentration of $1 \times 10^{4}$

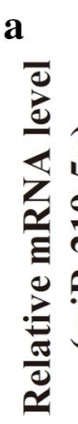

C

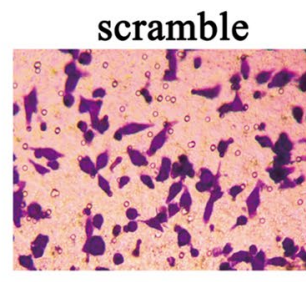

d

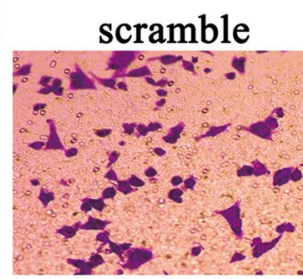

mimics

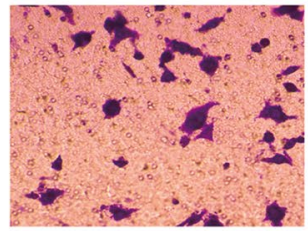

mimics

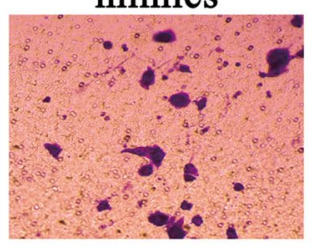

inhibitor

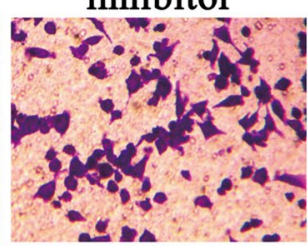

b

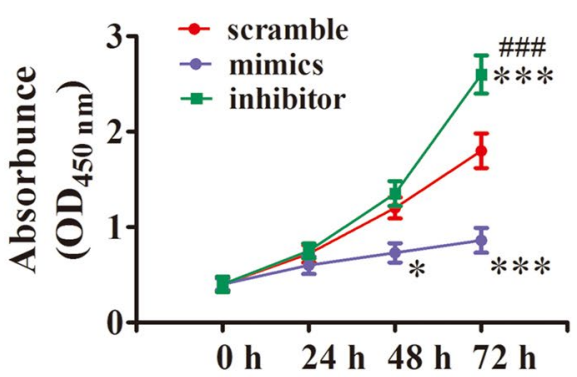

Oh 24 h 48 h 72 h
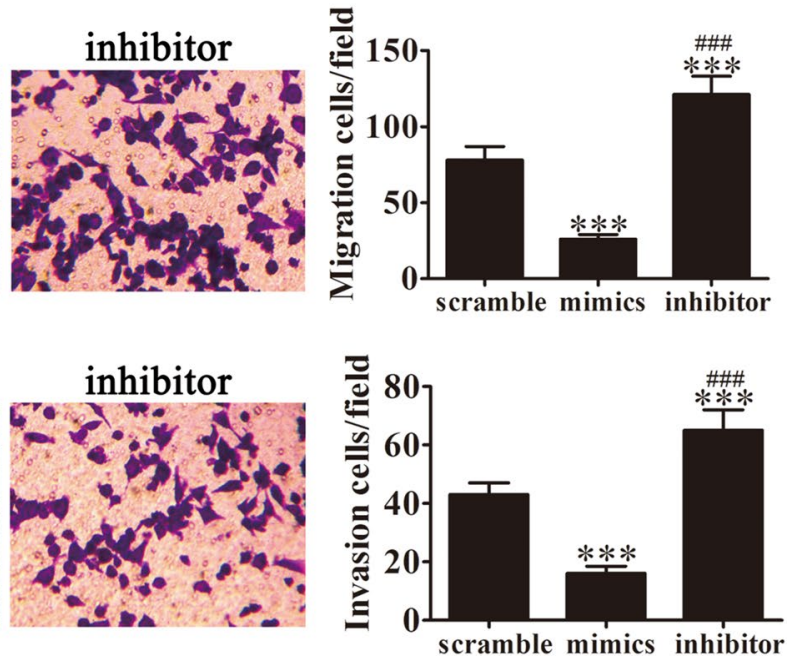

Fig. 1 miR-219-5p suppresses ovarian carcinoma cell proliferation, migration and invasion. a qRT-PCR showing expression of miR-219-5p in SKOV3 cells after treatment with the miR-219-5 $\mathrm{p}$ mimic, specific inhibitor or scramble (control) for $48 \mathrm{~h}$. Data are expressed as mean $\pm S D, n=5$. ${ }^{* * *} P<0.001$ versus scramble. $\mathbf{b}$ Measurement of SKOV3 cell proliferation with the CCK-8 assay. Data are expressed as mean $\pm S D, n=5$. ${ }^{*} P<0.05,{ }^{* * *} P<0.001$ versus scramble. ${ }^{\# \#} \mathrm{P}<0.001$ versus the mimic group. $\mathbf{c}, \mathbf{d}$ SKOV3 cell migration and invasion determined using the Transwe $\|^{\circledR}$ assay after treatment with the miR-219-5p mimic, specific inhibitor or scramble. Data are expressed as mean $\pm S D, n=5 .{ }^{* * *} P<0.001$ versus scramble. ${ }^{\# \#} P<0.001$ versus the mimic group 
cells cultured for different time periods $(0,24,48$, and 72 h). miR-219-5p overexpression led to significant suppression of proliferation of SKOV3 cells from $48 \mathrm{~h}$. In contrast, treatment with the miR-219-5p-specific inhibitor significantly promoted SKOV3 cell proliferation relative to the control group (Fig. 1b). In transwell migration (Fig. 1c) and invasion (Fig. 1d) assays, miR-219-5p overexpression led to significant suppression of migration and invasiveness of SKOV3 cells. Conversely, miR-219-5p inhibitor treatment promoted SKOV3 migration and invasiveness, compared with the scramble group. Our results collectively demonstrate that miR-219-5p exerts inhibitory effects on ovarian cancer cell proliferation, migration and invasion in vitro.
HMGA2 reverses the miR-219-5p-induced decrease in cell proliferation, migration and invasion

Previous studies have reported the involvement of HMGA2 in different steps of tumorigenesis. High levels of HMGA2 are reported in various cancer types, including ovarian cancer $[10,15]$. To determine whether HMGA2 is involved in miR-219-5p activity on tumor cell proliferation, an HMGA2 overexpression vector was constructed and successfully transfected into SKOV3 cells. Western blot results confirmed a significant increase in HMGA2 in SKOV3 cells after transfection with the overexpression vector (Fig. 2a). In previous studies, expression of miR-219-5p led to significant inhibition of SKOV3 cell proliferation. Notably, HMGA2 overexpression
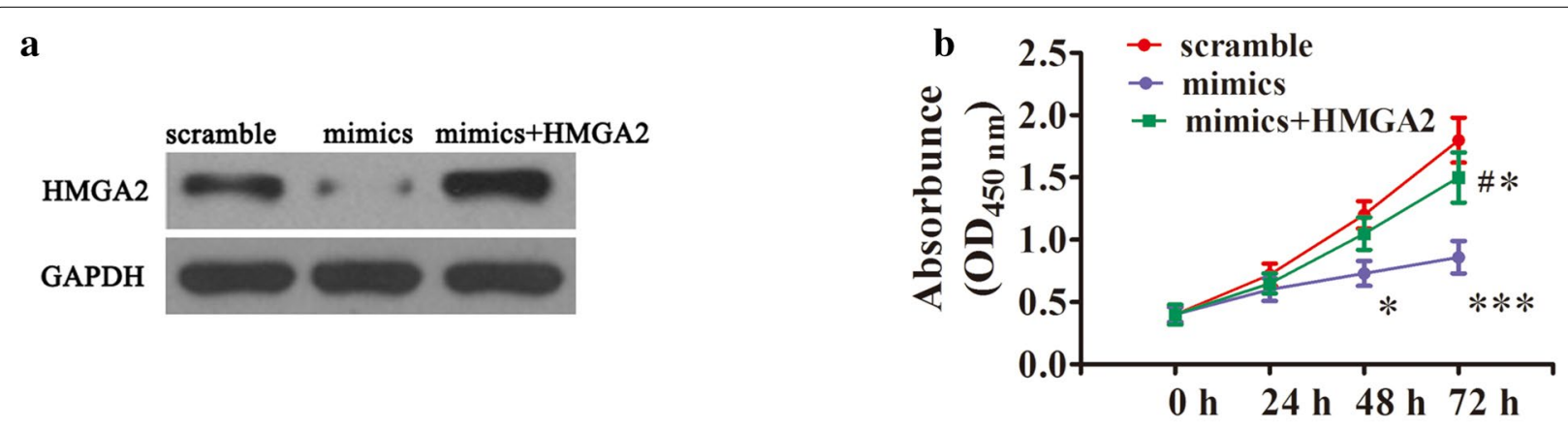

c
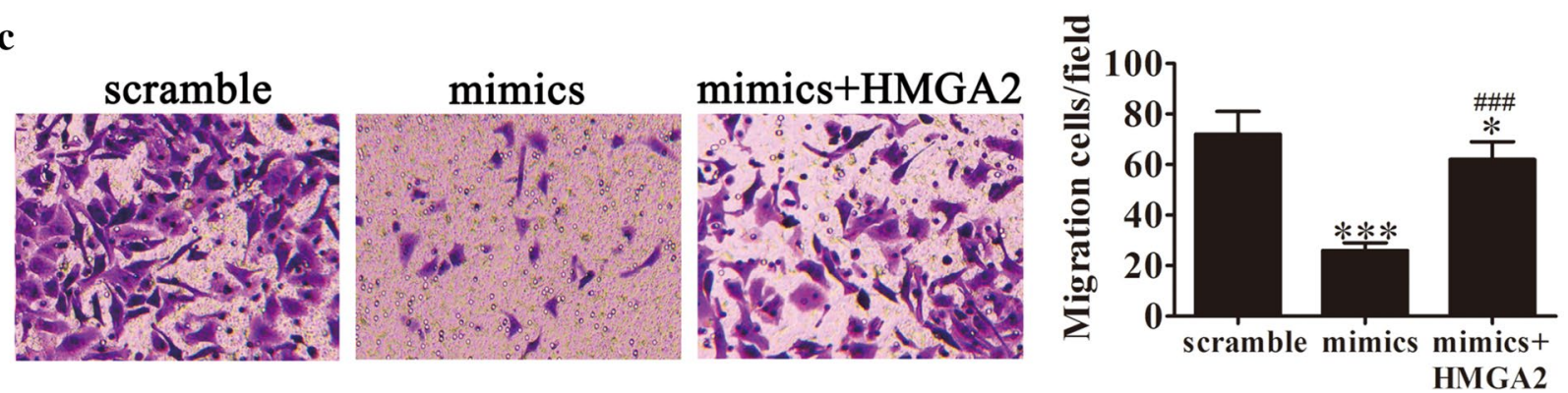

d
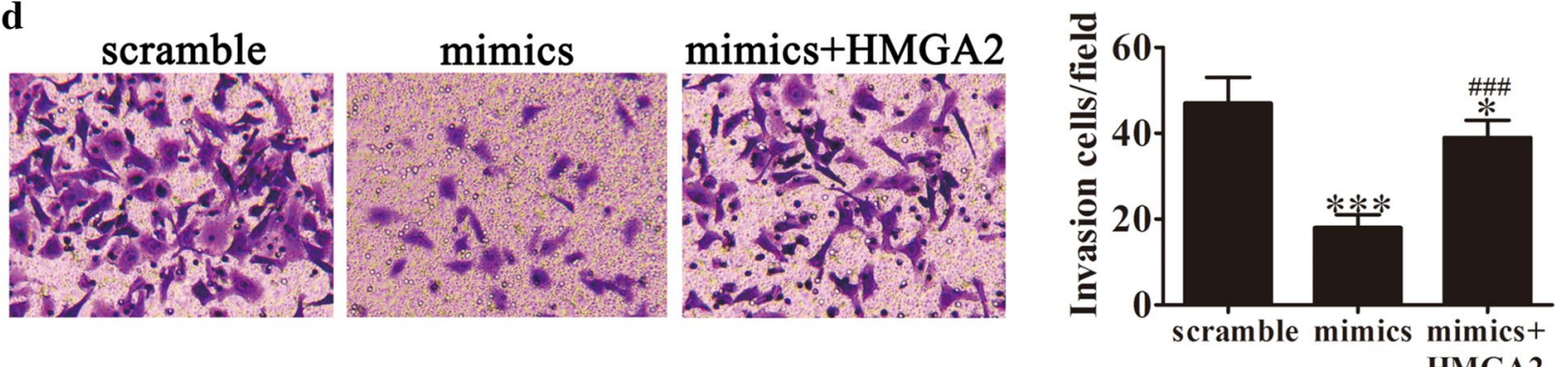

Fig. 2 HMGA2 overexpression suppresses miR-219-5p-induced cell proliferation, migration and invasion. a Western blot showing HMGA2 expression in SKOV3 cells after treatment with scramble, miR-219-5p mimic or HMGA2 (transfected with HMGA2 overexpression vector). $\mathbf{b}$ Overexpression of HMGA2 significantly reverses miR-219-5p-induced suppression of SKOV3 cell proliferation. Data are expressed as mean \pm SD, $n=5 .{ }^{*} P<0.05,{ }^{* *} P<0.001$ versus scramble. ${ }^{\#} P<0.05,{ }^{\# \#} P<0.001$ versus the mimic group. $\mathbf{c}, \mathbf{d}$ Cell migration and invasion of SKOV 3 determined using the Transwell ${ }^{\circledR}$ assay. Data are expressed as mean $\pm S D, n=5$. ${ }^{*} P<0.05$, ${ }^{* *} \mathrm{P}<0.001$ versus scramble. ${ }^{\# \# \#}<0.001$ versus the mimic group 
reversed the inhibitory effects of miR-219-5p on proliferation of SKOV3 cells (Fig. 2b). Transwell migration and invasion assays further showed that upregulation of HMGA2 significantly reverses miR-219-5p-induced inhibition of migration (Fig. 2c) and invasiveness (Fig. 2d), confirming that HMGA2 is involved in miR-219-5p mediated regulation of ovarian cancer cell growth.

\section{HMGA2 mRNA is a binding target of miR-219-5p}

Two major databases, TargetScan 7.0 and miRanda, were used to search for downstream targets of miR-219-5p, leading to the identification of HMGA2 as a potential target. Data from the luciferase reporter assay showed that the $3^{\prime}$ UTR of HMGA2 is a downstream binding target of miR-219-5p (Fig. 3a). Notably, miR-219-5p inhibited luciferase activity in constructs containing wild-type but not mutant $3^{\prime}$ UTR sites (Fig. 3b). Our data suggest that miR-219-5p interacts with the $3^{\prime} \mathrm{UTR}$ of HMGA2 and suppresses post-transcriptional HMGA2 expression. qRT-PCR analyses further confirmed that miR-219-5p overexpression is associated with inhibition of HMGA2 expression (Fig. 3c).

\section{miR-219-5p inhibits ovarian cancer growth in vivo}

In view of the finding that overexpression of miR-219-5p inhibits ovarian cancer cell proliferation in vitro, we further examined its antitumor effects in vivo. Control SKOV3 cells or those stably expressing miR-219-5p were subcutaneously inoculated into nude mice $(n=6$ for each group) and the sizes of SKOV3 tumors in mice measured using a caliper every 5 days. Tumor volume was significantly decreased in the group treated with the miR-219-5p mimic, compared with the control groups (Fig. 3d, e). Protein and mRNA expression of HMGA2 in xenograft tumors was determined via western blot (Fig. 3f) and qRT-PCR (Fig. 3g), respectively. HMGA2 was clearly downregulated in xenograft tumors from the miR-219-5p mimic group, compared to xenograft tumors of the control groups. The collective results showed that upregulation of miR-219-5p inhibits ovarian cancer

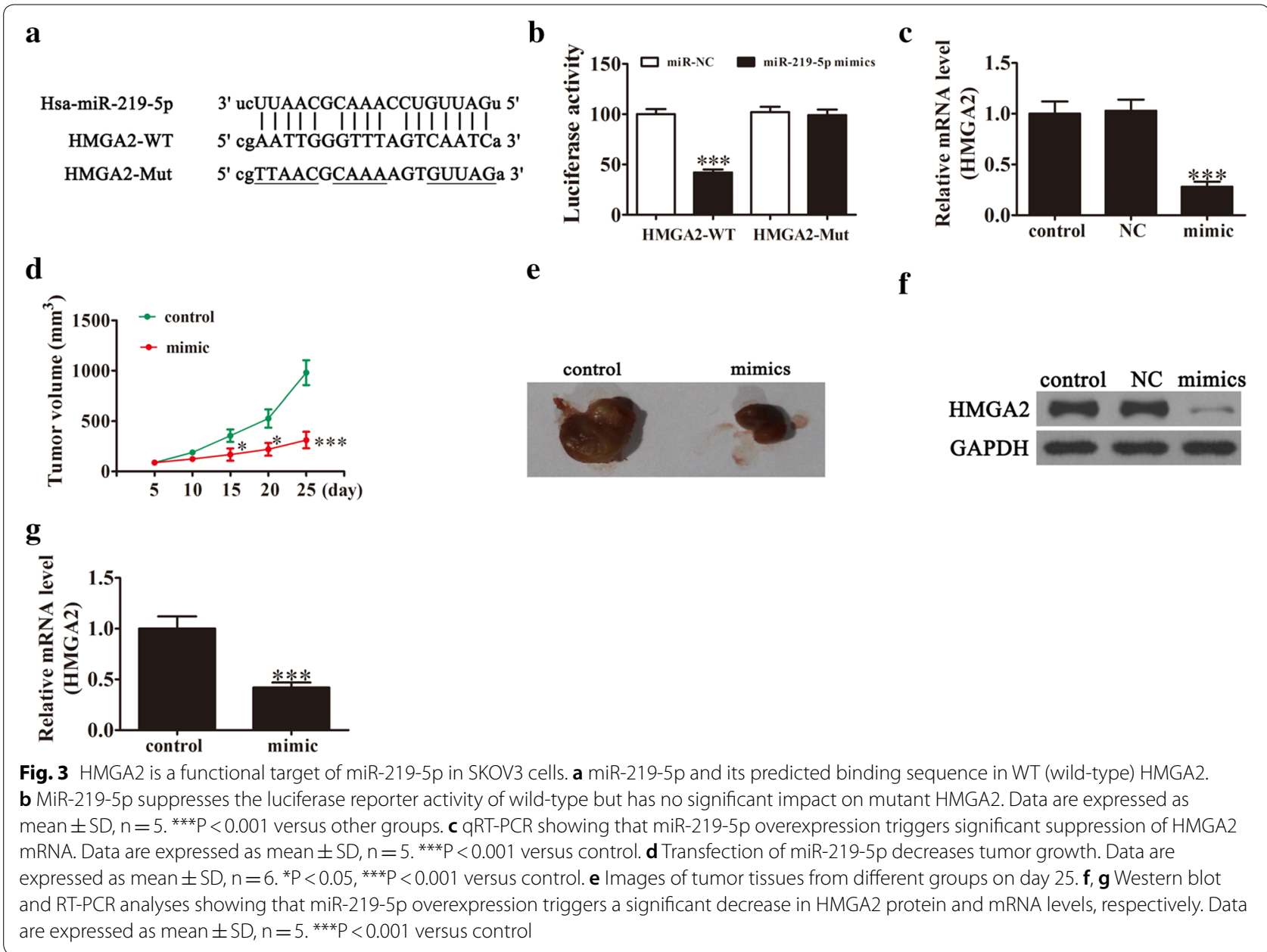


growth in vivo, which may be mediated via regulation of HMGA2 levels.

\section{Discussion}

Tumor development is a complex evolutionary process regulated by both protumor and antitumor elements [16]. Accumulating studies suggest that microRNAs play important roles in tumorigenesis through modulating target gene expression, including cell proliferation, differentiation, apoptosis and metastasis [17]. Abnormal expression and an antitumor role of miR-219-5p in a few cancer types has been documented in the literature. For example, miR-219-5p inhibits the proliferation, migration and invasion of epithelial ovarian cancer cells by targeting the Twist/Wnt/ $\beta$-catenin signaling pathway [18] and the growth and metastasis of malignant melanoma by targeting BCL-2 [14]. In the current study, miR-219-5p suppressed the proliferation, migration and invasion of ovarian cancer cells through suppression of HMGA2, both in vitro and in vivo.

HMGA2, a small, non-histone, chromatin-associated protein that plays a key role in tumorigenesis, acts as an transcription factor that regulates numerous genes [19]. HMGA2 overexpression has been frequently detected in several malignant neoplasms and inhibition of its expression shown to prevent tumor transformation in several cancer types, including thyroid cell [20], tongue squamous cell carcinoma [21] and breast cancer [22]. Data from this investigation clearly suggest that HMGA2 promotes ovarian cancer development. In our experiments, overexpression of HMGA2 reversed miR-219-5pinduced inhibition of ovarian cancer cell proliferation, migration and invasion. An earlier bi-fluorescein reporter experiment identified HMGA2 as a crucial downstream target of miR-219-5p. However, the precise mechanisms underlying HMGA2-mediated promotion of ovarian cancer cell growth processes require elucidation in future studies.

\section{Conclusions}

In conclusion, miR-219-5p inhibits cell proliferation, invasion and migration of ovarian cancer cells through interactions with the $3^{\prime}$-UTR of HMGA2. This miRNA may provide a novel diagnostic and therapeutic option for patients with ovarian cancer.

\section{Abbreviations \\ HMGA2: high-mobility gene group A2; miRNA: microRNA; 3'UTR: 3'-untrans- lated region; FBS: fetal bovine serum.}

\section{Authors' contributions}

Conception and design of entire study: FX, ZS. Analysis and interpretation of data: YH. Drafted the article: FX. Revised the manuscript critically for content: ZS. All authors read and approved the final manuscript.
Acknowledgements Not applicable.

Competing interests

The authors declare that they have no competing interests.

Availability of data and materials

All data generated or analysed during this study are included in this article.

Consent for publication

Not applicable.

Ethics approval and consent to participate

This study was approved by the ethics review board of Shanghai Tenth People's Hospital, Shanghai, China.

\section{Funding}

This study was not supported by funding from any source.

\section{Publisher's Note}

Springer Nature remains neutral with regard to jurisdictional claims in published maps and institutional affiliations.

Received: 30 May 2018 Accepted: 18 November 2018

Published online: 24 November 2018

\section{References}

1. Galdiero F, Romano A, Pasquinelli R, Pignata S, Greggi S, Vuttariello E, Bello AM, Calise C, Scaffa C, Pisano C, Losito NS, Fusco A, Califano D, Chiappetta G. Detection of high mobility group a2 specific mrna in the plasma of patients affected by epithelial ovarian cancer. Oncotarget. 2015;6:19328-35

2. Siegel R, Naishadham D, Jemal A. Cancer statistics, 2012. CA Cancer J Clin. 2012;62:10-29.

3. Hutchinson L. Targeted therapies: Solo2 confirms olaparib maintenance in ovarian cancer. Nat Rev Clin Oncol. 2017;14:586-7.

4. Fusco A, Fedele M. Roles of hmga proteins in cancer. Nat Rev Cancer. 2007;7:899-910

5. Hammond SM, Sharpless NE. Hmga2, micrornas, and stem cell aging. Cell. 2008;135:1013-6.

6. Wu J, Zhang S, Shan J, Hu Z, Liu X, Chen L, Ren X, Yao L, Sheng H, Li L, Ann $D$, Yen $Y$, Wang J, Wang $X$. Elevated hmga2 expression is associated with cancer aggressiveness and predicts poor outcome in breast cancer. Cancer Lett. 2016:376:284-92.

7. Palumbo A Jr, Da Costa NM, Esposito F, De Martino M, D'Angelo D, de Sousa VP, Martins I, Nasciutti LE, Fusco A, Ribeiro Pinto LF. Hmga2 overexpression plays a critical role in the progression of esophageal squamous carcinoma. Oncotarget. 2016;7:25872-84.

8. Fan C, Lin Y, Mao Y, Huang Z, Liu AY, Ma H, Yu D, Maitikabili A, Xiao H, Zhang C, Liu F, Luo Q, Ouyang G. Microrna-543 suppresses colorectal cancer growth and metastasis by targeting kras, mta1 and hmga2. Oncotarget. 2016;7:21825-39.

9. Wu J, Wang Y, Xu X, Cao H, Sahengbieke S, Sheng H, Huang Q, Lai M. Transcriptional activation of fn 1 and il 11 by hmga 2 promotes the malignant behavior of colorectal cancer. Carcinogenesis. 2016;37:511-21.

10. Agostini A, Brunetti M, Davidson B, Trope CG, Heim S, Panagopoulos I, Micci F. Genomic imbalances are involved in mir-30c and let-7a deregulation in ovarian tumors: implications for hmga2 expression. Oncotarget. 2017:8:21554-60

11. Tutar Y. Mirna and cancer; computational and experimental approaches. Curr Pharm Biotechnol. 2014;15:429.

12. Trang P, Weidhaas JB, Slack FJ. Micrornas as potential cancer therapeutics. Oncogene. 2008;27(Suppl 2):S52-7. 
13. Cheng J, Deng R, Zhang P, Wu C, Wu K, Shi L, Liu X, Bai J, Deng M, Shuai X, Gao J, Wang G, Tao K. Mir-219-5p plays a tumor suppressive role in colon cancer by targeting oncogene sall4. Oncol Rep. 2015;34:1923-32.

14. Long J, Menggen Q, Wuren Q, Shi Q, Pi X. Mir-219-5p inhibits the growth and metastasis of malignant melanoma by targeting bcl-2. Biomed Res Int. 2017;2017:9032502.

15. Wu J, Lai M, Shao C, Wang J, Wei JJ. Stc2 overexpression mediated by hmga2 is a biomarker for aggressiveness of high-grade serous ovarian cancer. Oncol Rep. 2015:34:1494-502.

16. McGranahan N, Swanton C. Clonal heterogeneity and tumor evolution: past, present, and the future. Cell. 2017;168:613-28.

17. Qiu L, Tang Q, Li G, Chen K. Long non-coding rnas as biomarkers and therapeutic targets: recent insights into hepatocellular carcinoma. Life Sci. 2017;191:273-82

18. Wei C, Zhang X, He S, Liu B, Han H, Sun X. Microrna-219-5p inhibits the proliferation, migration, and invasion of epithelial ovarian cancer cells by targeting the twist/wnt/beta-catenin signaling pathway. Gene. 2017;637:25-32.

19. Wu J, Wei JJ. Hmga2 and high-grade serous ovarian carcinoma. J Mol Med (Berl). 2013;91:1155-65.

20. Califano D, Pignata S, Losito NS, Ottaiano A, Greggi S, De Simone V, Cecere S, Aiello C, Esposito F, Fusco A, Chiappetta G. High hmga2 expression and high body mass index negatively affect the prognosis of patients with ovarian cancer. J Cell Physiol. 2014;229:53-9.

21. Zhang H, Tang Z, Deng C, He Y, Wu F, Liu O, Hu C. Hmga2 is associated with the aggressiveness of tongue squamous cell carcinoma. Oral Dis. 2017;23:255-64.

22. Zou Q, Wu H, Fu F, Yi W, Pei L, Zhou M. Rkip suppresses the proliferation and metastasis of breast cancer cell lines through up-regulation of mir185 targeting hmga2. Arch Biochem Biophys. 2016;610:25-32.
Ready to submit your research? Choose BMC and benefit from:

- fast, convenient online submission

- thorough peer review by experienced researchers in your field

- rapid publication on acceptance

- support for research data, including large and complex data types

- gold Open Access which fosters wider collaboration and increased citations

- maximum visibility for your research: over $100 \mathrm{M}$ website views per year

At BMC, research is always in progress.

Learn more biomedcentral.com/submissions 